\title{
Inhibition of the Sonic Hedgehog Pathway by Cyclopamine or GLI1 siRNA Reduces In Vivo Tumorigenesis of Human Medulloblastoma Cells Xenotransplanted to Immunodefi- cient Nude Mice
}

\section{Javier de la Rosa ${ }^{1,2}$, Mónica Sánchez', Mónica Enguita-Germán², Ricardo García-López', Paula Schiapparelli², Mehdi H Shahï, Bárbara Meléndez ${ }^{4}$, Juan A Rey ${ }^{5}$, Miguel A Idoate ${ }^{6}$ and Javier S Castresana ${ }^{1,2 *}$}

${ }^{1}$ Department of Biochemistry and Genetics, University of Navarra, Spain

${ }^{2}$ Brain Tumor Biology Unit, University of Navarra, Spain

${ }^{3}$ Faculty of Medicine, Interdisciplinary Brain Research Centre, Aligarh Muslim University, India

${ }^{4}$ Molecular Pathology Research Unit, Virgen de la Salud Hospital, Spain

5/diPaz Research Unit, La Paz University Hospital, Spain

${ }^{6}$ Department of Pathology, University of Navarra, Spain

\begin{abstract}
Medulloblastoma is an aggressive tumor; grade IV of the WHO classification that develops in the cerebellum, mostly linked to infancy and adolescence. It can be classified histologically and molecularly in different subtypes. Morphologically it can be divided into classical, desmoplastic/nodular, anaplastic, and large cell medulloblastoma. Molecularly, there are four groups: Wnt, Shh (sonic hedgehog), group 3 (mainly linked to MYC amplification), and group 4 (for unclassified tumors). Our work tries to prove the inhibition of the Shh pathway in two medulloblastoma cell lines: DAOY, a desmoplastic cerebellar medulloblastoma cell line of the Shh molecular group; and D283 Med, derived from a metastasis to peritoneum, possibly corresponding to an aggressive group 3 medulloblastoma. Cyclopamine, an inhibitor of the SMO oncogenic protein, and GLI1 siRNA were used as inhibitory agents of the Shh pathway in the two cell lines. For the in vivo assay, for each cell line, each experimental group consisted of 6 mice injected subcutaneously with control cells on the right flank, and cells treated with cyclopamine or GLI1 siRNA on the left flank. In the cell lines studied, cyclopamine showed a high inhibitory growth of subcutaneous tumors in the D283 Med and DAOY lines. The siRNA treatment, however, was only effective in the D283 Med cell line.
\end{abstract}

Keywords

Medulloblastoma, Sonic hedgehog, Cyclopamine, GLI1 siRNA, Xenotransplantation

\section{Introduction}

Medulloblastoma, a tumor of the cerebellum, is one of the most frequent pediatric tumors [1], usually appearing in infancy and adolescence, and very rarely in adults. Some genetic syndromes, like Gorling, Turcot, and Li-Fraumeni, predispose to the development of medulloblastoma [2]. Surgery, radiotherapy and chemotherapy are used against this tumor, but patients suffer devastating neurocognitive sequelae due to the aggressiveness of these treatments [3]. For this reason, understanding the
*Corresponding author: Javier S Castresana, Department of Biochemistry and Genetics, University of Navarra School of Sciences, Irunlarrea 1, 31008 Pamplona, Spain, Tel: +34948-425600, Ext: 806486, E-mail: jscastresana@unav.es

Received: June 16, 2017; Accepted: July 14, 2017; Published online: July 19, 2017

Citation: de la Rosa J, Sánchez M, Enguita-Germán M, et al. (2017) Inhibition of the Sonic Hedgehog Pathway by Cyclopamine or GLI1 siRNA Reduces In Vivo Tumorigenesis of Human Medulloblastoma Cells Xenotransplanted to Immunodeficient Nude Mice. Advances Transl Med Res 1(1):1-5

Copyright: (C) 2017 de la Rosa J, et al. This is an open-access article distributed under the terms of the Creative Commons Attribution License, which permits unrestricted use, distribution, and reproduction in any medium, provided the original author and source are credited. 
molecular mechanisms of the pathogenesis of medulloblastoma and designing new approaches to treat this disease are important in biomedical research [1].

This embryonic tumor is classified in four histological sub types according to the World Health Organization: classical, desmoplastic/nodular, anaplastic, and large cell medulloblastomas $[4,5]$. Moreover, four molecular subtypes have recently been introduced: Wnt, Shh (sonic hedgehog), group 3 (usually presenting MYC amplification), and group 4 (linked to isochromosome $17 \mathrm{q}$, and mostly including those medulloblastomas not clearly belonging to other groups) [6].

The subgroup of Shh medulloblastomas are produced by alterations in the sonic hedgehog signaling pathway. In the absence of the Shh ligand, PTCH inhibits the function of SMO, thereby inhibiting GLI1 activation [7]. In the presence of the Shh ligand, PTCH eliminates its inhibitory effect on SMO, which will promote activation of GLI1, its translocation to the nucleus, and thereby stimulation of transcription of its target genes such as GLI1, BCL-2, MYCN, BMI1, CCND2, PTCH1 and HHIP. Aberrant and altered signaling of the Shh pathway leads to certain types of cancers and tumors [8].

A possible therapeutic strategy for the treatment of medulloblastoma might be the use of inhibitors of the Shh pathway such as cyclopamine that directly inhibits SMO function. Another possibility would be an approach to knock-down GLI1 by siRNA [1]. We have used the two approaches in two different medulloblastoma cell lines: DAOY, a desmoplastic cerebellar medulloblastoma cell line of the Shh molecular group; and D283 Med, derived from a metastasis to peritoneum, possibly corresponding to an aggressive group 3 medulloblastoma.

Previous results from our group demonstrated that both cell lines express several genes related to the Shh pathway (SHH, PTCH, SMO, GLI1, GLI2, GLI3, MYCN, BMI1, HHIP and SUFU) at very similar levels [9], determined by RT-PCR experiments. SHH level of expression was a bit smaller in D283 Med than in DAOY, although SUFU slightly showed the opposite results. MYCN expression was similar in both cell lines, but none of them presented MYCN gene amplification (ratio above 10) assayed by qPCR, although DAOY presented an amplification ratio of 6.4, and D283 Med a ratio below 3 (unpublished results). We treated both cell lines with cyclopamine to inhibit the Shh pathway [9]. PTCH and GLI relative expression decreased after cyclopamine treatment, more noticeable in DAOY (60\% decrease) than in D283 Med cells (20\% decay). Respect to cell survival, 20\% DAOY cells and 50\% D283 Med cells survived the cyclopamine treatment. Finally, an in vitro tumorigenicity test, based on a colony forming assay in soft agar showed $80 \%$ and $50 \%$ decay in the number of colonies in DAOY and D283 Med treated cell lines respectively, compared to controls. These results show that DAOY, a Shh medulloblastoma cell line responds better to treatment with cyclopamine than D283 Med, as DAOY treated cells presented a higher decrease in PTCH and GLI expression, a lower cell survival, and a higher decay in colony formation in soft agar.

Next, we decided to further explore the inhibition of the Shh pathway in the DAOY cell line with a double method of treatment: cyclopamine (to inhibit SMO function, upstream of GLI), versus GLI1 siRNA (to inhibit GLI1 itself) [1]. Cyclopamine inhibited GLI1 expression in DAOY cells by $85 \%$, and siRNA inhibited GLI by $95 \%$. The ability to form $2 \mathrm{D}$ colonies on cell plates suffered a $70 \%$ reduction under both conditions of treatment, while the ability to form 3D colonies in soft agar was reduced in a higher way (90\% reduction with cyclopamine, and 95\% reduction with GLI1 siRNA treatment, respect to controls). Finally, a cell migration wound (or scratching) assay was performed in the DAOY cell line, and showed that cell migration was reduced under both treatments compared to controls. These results show that cyclopamine and GLI1 siRNA both are relevant possibilities to in vitro treat Shh medulloblastoma cells: the reduction in 3D colonies in soft agar was even bigger than the reduction in $2 \mathrm{D}$ colonies, which might indicate that our treatments can reduce the cancer stem cell compartment, as 3D colonies are representative of these initiating and cancer-maintaining cell subpopulations. Even more, we have demonstrated that inhibition of Shh pathway by cyclopamine reduces the CD133+/CD15+ cell compartment and the in vitro tumorigenic capability of neuroblastoma cells [10]. And that CD133+ cells from medulloblastoma and PNET cell lines are more resistant to cyclopamine inhibition of the Shh signaling pathway than CD133- cells [9], which is in agreement with the theory that cancer stem cells are resistant to chemotherapy.

The present work promotes the idea of an in vivo experiment to further investigate the possibility of inhibiting the Shh pathway by two methods in medulloblastoma cell lines. In our experience $[1,9,10]$, both cyclopamine and GLI1 siRNA appropriately behave in vitro assays, inhibiting the Shh pathway and reducing the cancer stem cells compartment. We treated the DAOY and D283 Med cell lines with cyclopamine or GLI1 siRNA and xenotransplanted them to immunodeficient nude mice for testing the capability of the treatments to reduce in vivo tumorigenesis respect to controls.

\section{Materials and Methods}

\section{Cell lines}

Medulloblastoma cell lines DAOY and D283 Med, obtained from the American Type Culture Collection (ATCC, Manassas, VA, USA), were used. The lines were cultured 
using RPMI L-Glutamax medium (Gibco, Gaithersburg, MD, USA) supplemented with $10 \%$ Fetal Bovine Serum (FBS) (Gibco, Invitrogen, Carlsbad, CA, USA), 4\% non essential amino acids (Lonza, Verviers, Belgium), 1\% penicillin/streptomycin (Gibco, Invitrogen, Carlsbad, CA, USA) and $0.1 \%$ amphotericin B (Gibco, Invitrogen, Carlsbad, CA, USA). All cells used were maintained under normoxia conditions at $37^{\circ} \mathrm{C}$ with a humid $5 \% \mathrm{CO}_{2}$ atmosphere. Subcultures were performed at $80 \%$ confluence using trypsin/EDTA (Gibco, Invitrogen, Carlsbad, CA, USA) after pre-washing with phosphate buffered saline (Gibco, Invitrogen, Carlsbad, CA, USA). Both cell lines had previously been studied for expression of regulators of the Shh pathway by RT-PCR (SHH, PTCH, SMO, GLI1, GLI2, GLI3, MYCN. BMI1, HHIP, and SUFU) [9].

\section{Treatment of cell lines with cyclopamine}

Cyclopamine treatments were performed using 16 $\mu \mathrm{M}$ cyclopamine tartrate dissolved in DMSO for DAOY and $20 \mu \mathrm{M}$ for D283 Med [11]. For this purpose, the cells were incubated with low concentration of FBS (2.5\%) supplemented with cyclopamine at these concentrations for $48 \mathrm{~h}$. Control cells received a treatment with the indicated amount of DMSO. After $48 \mathrm{~h}$, the surviving cells were trypsinized for inoculation in mice.

\section{Treatment of cell lines with GLI1 siRNA}

Previously to this experiment, we did a GLI1 knockdown experiment [1] to be sure that GLI1 expression is highly reduced due to treatment. Also, the next genes were assayed for qRT-PCR expression after treatment [1]: PTCH1, CCND2, PAX6, Plakoglobin (gamma-catenin), NKX2-2, BMI-1, SMO, SUFU, GLI3, MYCN, NOTCH1 and NOTCH2. Cells were plated in 6-well plates, and incubated with RPMI supplemented with $2 \%$ FBS. A pool of siRNAs against GLI1 from StealthTM (Invitrogen, Carlsbad, CA, USA) was used in order to ensure GLI1 complete inhibition.

\section{GLI1 siRNA}

GCACAUACCUGCUUCGGGCAAGAUAU (GLI-HSS104170)

AUAUCUUGCCCGAAGCAGGUAGUGC

(GLI-HSS104170)

CAGCUUGGACGAGGGACCUUGCAUU

(GLI-HSS178441)

AAUGCAAGGUCCCUCGUCCAAGCUG

(GLI-HSS178441)

GGGAUGGCUGCAGCCAGGAAUUUGA

(GLI-HSS178442)

UCAAAUUCCUGGCUGCAGCCAUCCC

\section{(GLI-HSS178442)}

The GLI1 siRNAs have been previously checked for their efficiency in specifically silencing GLI1 expression [1]. We delivered siRNAs against GLI1 $(60 \mathrm{nM} /$ well $)$ and its negative control to the cell lines using Lipofectamine 2000 as a transfection agent and Opti-MEM I reduced serum medium. After $48 \mathrm{~h}$, the surviving cells were trypsinized for inoculation in mice.

\section{Xenotransplantation to nude mice}

For xenotransplantation of human medulloblastoma cells to immunodeficient athymic nude mice, initially, a pilot trial was conducted for testing the ability of the DAOY and D283 Med cell lines to generate subcutaneous tumors in nude mice. We wanted the xenotransplant to produce a tumor that could grow in a continued way and not in an excessively accelerated way along time. For each cell line, 2, 4, 8 and 10 million untreated cells were injected subcutaneously into the flanks of the mice, in order to decide the appropriate number of cells for the experiment to be done with the treated cells. Each cell number was injected twice, into two distinct mice. The pilot experiment allowed the choice of the following cell numbers for the treated cell xenograft experiment: 10 million cells for D283 Med, and 4 million cells for DAOY.

For the in vivo assay, DAOY and D283 Med cells were pre-treated with DMSO, cyclopamine, or GLI1 siRNA for $48 \mathrm{~h}$. For each cell line, each experimental group consisted of 6 mice injected subcutaneously with control cells on the right flank, and cells treated with cyclopamine or siRNA on the left flank. The cells were resuspended in $200 \mu$ of DPBS, kept on ice, and injected into 6 -week-old female nude mice that were anesthetized with $3-4 \%$ isoflurane (Abbot S.A.) in $100 \%$ oxygen. Tumor growth was measured every 3-4 days, determining the largest longitudinal (long) and transverse (wide) diameter with a Vernier precision gauge. Each mouse was sacrificed when the sum of the diameter of the tumors on its flanks reached $17 \mathrm{~mm} .10$ million D283 Med cells, and 4 million DAOY cells were injected into each flank.

\section{Results and Discussion}

In the cell lines studied, cyclopamine showed a high inhibitory growth of subcutaneous tumors in the D283 Med and DAOY lines $(\mathrm{p}<0.0001)$. The siRNA treatment, however, was only effective in the D283 Med cell line $(\mathrm{p}<0.0001)$ (Figure 1). Our work consisted of an in vivo tumorigenesis assay, by xenotransplantation of human medulloblastoma cell lines previously treated with cyclopamine or with GLI1 siRNA (and those not treated as a control) to immunodeficient nude mice. The study was performed with DAOY and D283 Med lines. Cy- 
Citation: de la Rosa J, Sánchez M, Enguita-Germán M, et al. (2017) Inhibition of the Sonic Hedgehog Pathway by Cyclopamine or GLI1 siRNA Reduces In Vivo Tumorigenesis of Human Medulloblastoma Cells Xenotransplanted to Immunodeficient Nude Mice. Advances Transl Med Res 1(1):1-5
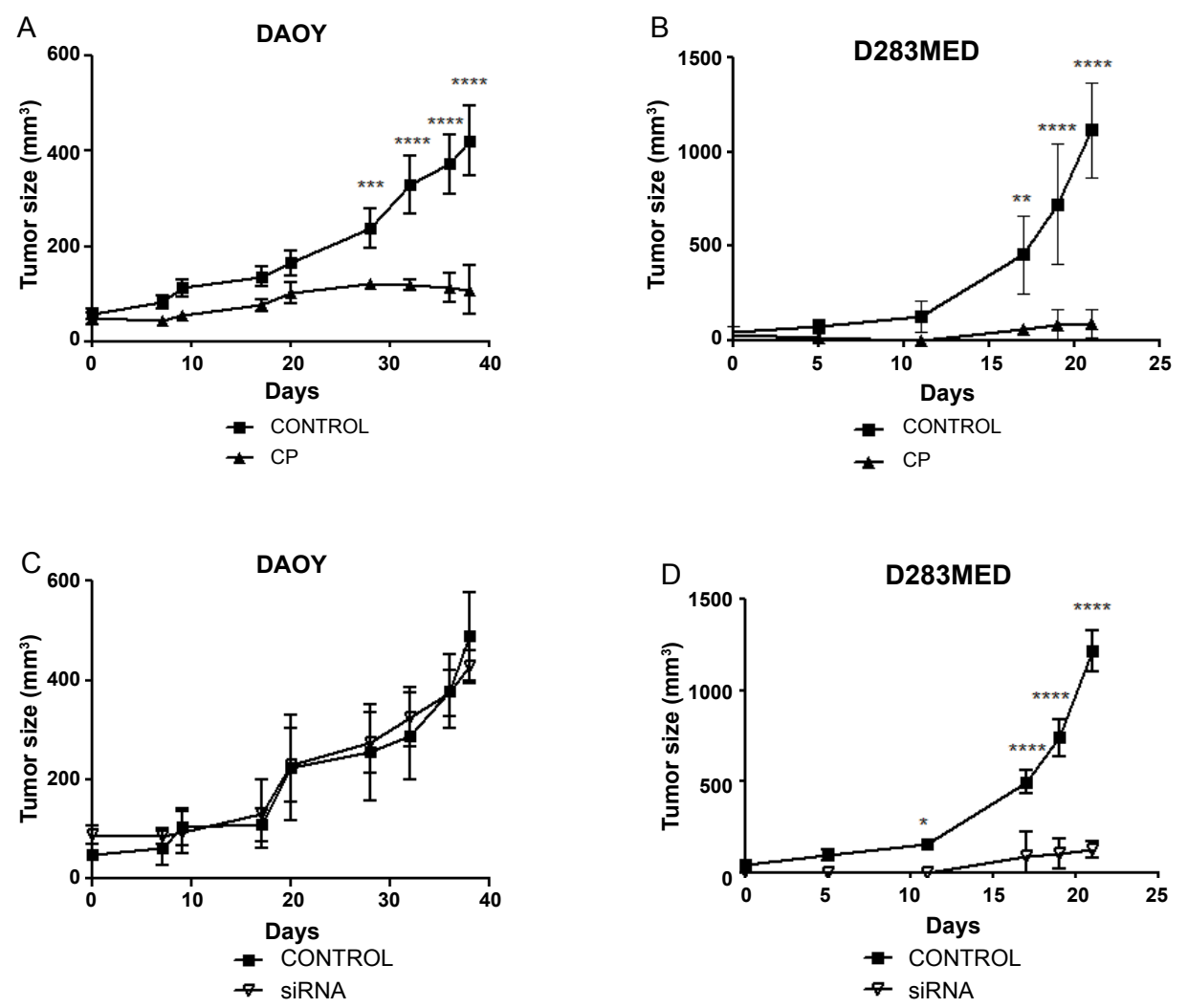

$E$

\section{DAOY}

\section{D283MED}

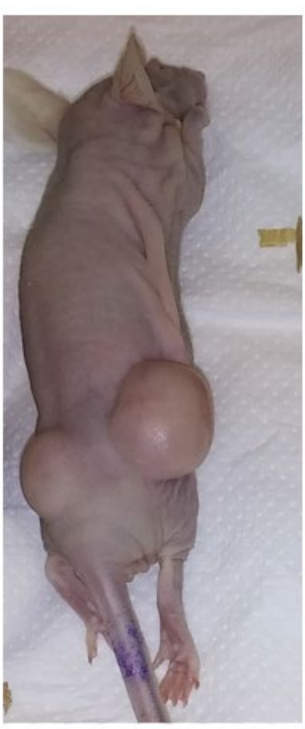

$\mathrm{CP}$

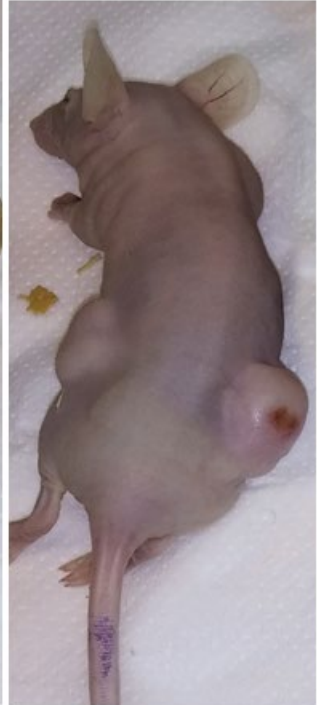

SiRNA

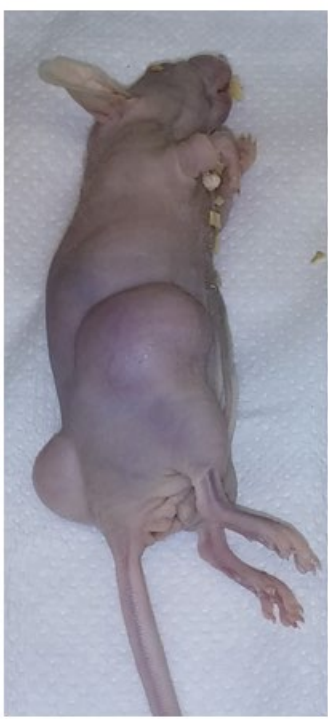

$\mathrm{CP}$

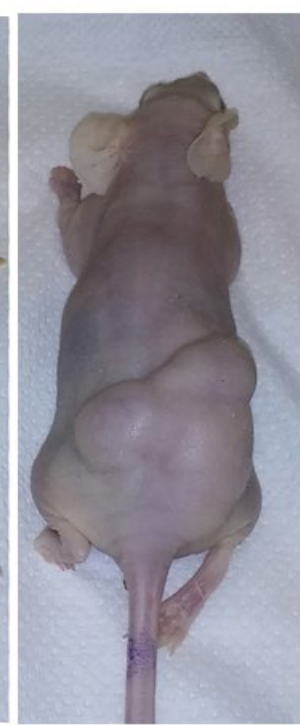

SiRNA

Figure 1: Growth of in vivo xenotransplantation of human medulloblastoma cells to immunodeficient nude mice. A) Inhibition of tumor growth by treatment with cyclopamine in DAOY cells. B) Inhibition of tumor growth by treatment with cyclopamine in D283Med cells. C) Inhibition of tumor growth by GLI1 siRNA treatment in DAOY cells. D) Inhibition of tumor growth by GLI1 siRNA treatment in D283Med cells. CP: cyclopamine. ${ }^{*}: P<0.05,{ }^{* *}: p<0.01,{ }^{* * *}: p<0.001,{ }^{* * * *}: p<0.0001$, according to ANOVA and multiple comparison test of Sidak.

E) Representative pictures of mice with xenografts of DAOY or D283Med treated cells. The xenotransplantation of the right flank of the mouse corresponds to the control treatment, while those of the left flank correspond to the treatments with cyclopamine $(\mathrm{CP})$ or siRNA against GLI1. 
Citation: de la Rosa J, Sánchez M, Enguita-Germán M, et al. (2017) Inhibition of the Sonic Hedgehog Pathway by Cyclopamine or GLI1 siRNA Reduces In Vivo Tumorigenesis of Human Medulloblastoma Cells Xenotransplanted to Immunodeficient Nude Mice. Advances Transl Med Res 1(1):1-5

clopamine could effectively inhibit the tumor growth of DAOY and D283 Med lines alike. The D283 Med cell line showed greater inhibition of tumor growth after siRNA treatment than DAOY. It might probably be due to baseline GLI1 expression differences in each cell line. Taking into account that the DAOY line is prototypical of the Shh type of medulloblastomas, it is clear that cyclopamine treatment can be very effective against it. The D283 Med line is more similar, on the contrary, to medulloblastoma groups not well known, but more aggressive than the Shh and Wnt groups, the so-called group 3 (associated with over expression of MYC) and group 4 (which accumulates those medulloblastomas not well classified in the preceding groups). Moreover, DAOY, besides presenting canonical activation of the Shh pathway, could be associated with non-canonical activation of this pathway, producing an increase of GLI1 not necessarily due to an increase in SMO activity, but to other regulators of cell signaling, external to the Shh pathway, such as TGF beta, PI3K/Akt, KRAS, MYC, or WNT/beta-catenin. If D283 Med is an example of group 3 medulloblastoma, with over expression of MYC, it will also over express GLI1, but perhaps in lesser amounts than the DAOY cell line through the canonical Shh pathway or through both the canonical and the non-canonical pathways. In such a case, if D283 Med produced lower GLI1 levels than DAOY, it would be plausible to think that siRNA against GLI1 would have greater effectiveness in D283 Med than in DAOY, as it indeed appears in our results.

As a conclusion of our results of this work, and after accumulating the experience of in vitro treatments against Shh $[1,9,10]$, we may say that inhibition of the Shh pathway seems to be more efficient if an approach to directly inhibit GLI1 is applied, such as GLI1 siRNA, at least in the experiments in vitro. Quite the contrary, xenotransplantation studies to nude mice demonstrate that cyclopamine reduces in vivo tumorigenesis in the two cell lines, while GLI1 siRNA is effective only against the D283 Med cell line, and not against DAOY, which might indicate that Shh dependent medulloblastomas (like DAOY) should better be treated in vivo by cyclopamine, an SMO inhibitor, instead of by GLI1 siRNA knock-down.

Apart from our data, one may have into account that several other SMO inhibitors are being studied. Cyclopamine, the first developed inhibitor of SMO, is a teratogen that produces holoprosencephaly and cyclopia. Even, it cannot be administered orally, and has several side effects, making it impossible as a candidate to be used in humans [12]. Vismodegib is the first FDA-approved SMO inhibitor [13], in 2012, for the treatment of basal cell carcinoma and has been subsequently studied against colon [14], pancreatic [15], and medulloblasto- ma tumors [16]. In patients suffering from resistance to treatment [17-20], sonidegib, the second inhibitor of SMO approved by the FDA in 2015 [21], is used [22-24]. Other possible inhibitors against SMO, subjected to research, and possibly used in resistance to vismodegib and sonidegib [25], are natural products such as chalcone 12 [26], analogs of vitamin D3 [27], or itraconazole, the new inhibitor that might act against mutated SMO [28]. The appropriate use of all these compounds constitutes a new research area that requires basic knowledge of the Shh pathway regulation.

\section{Acknowledgements}

This research was supported by a grant from Fundación Universitaria de Navarra, Pamplona, Spain. We thank Alejandro Urdiciain, MSc, for artwork with GraphPad.

\section{References}

1. García-López R, Vera-Cano B, Vacas-Oleas A, et al. (2013) Sonic hedgehog inhibition reduces in vitro tumorigenesis and alters expression of Gli1-target genes in a desmoplastic medulloblastoma cell line. Journal of Cancer Research \& Therapy 1: 11-23.

2. Taylor MD, Mainprize TG, Rutka JT (2000) Molecular insight into medulloblastoma and central nervous system primitive neuroectodermal tumor biology from hereditary syndromes: a review. Neurosurgery 47: 888-901.

3. Rutkowski S, von Hoff K, Emser A, et al. (2010) Survival and prognostic factors of early childhood medulloblastoma: an international meta-analysis. J Clin Oncol 28: 4961-4968.

4. Louis DN, Ohgaki H, Wiestler OD, et al. (2007) The 2007 WHO classification of tumours of the central nervous system. Acta Neuropathol 114: 97-109.

5. Louis DN, Perry A, Reifenberger G, et al. (2016) The 2016 World Health Organization Classification of Tumors of the Central Nervous System: a summary. Acta Neuropathol 131: 803-820.

6. Taylor MD, Northcott PA, Korshunov A, et al. (2012) Molecular subgroups of medulloblastoma: the current consensus. Acta Neuropathol 123: 465-472.

7. Shahi MH, Rey JA, Castresana JS (2012) The sonic hedgehog-GLI1 signaling pathway in brain tumor development. Expert Opin Ther Targets 16: 1227-1238.

8. Abidi A (2014) Hedgehog signaling pathway: a novel target for cancer therapy: vismodegib, a promising therapeutic option in treatment of basal cell carcinomas. Indian J Pharmacol 46: 3-12.

9. Enguita-German M, Schiapparelli P, Rey JA, et al. (2010) CD133+ cells from medulloblastoma and PNET cell lines are more resistant to cyclopamine inhibition of the sonic hedgehog signaling pathway than CD133- cells. Tumour Biol 31: 381-390.

10. Schiapparelli P, Shahi MH, Enguita-German M, et al. (2011) Inhibition of the sonic hedgehog pathway by cyplopamine reduces the CD133+/CD15+ cell compartment and the in vitro tumorigenic capability of neuroblastoma cells. Cancer Lett 310: 222-231. 
Citation: de la Rosa J, Sánchez M, Enguita-Germán M, et al. (2017) Inhibition of the Sonic Hedgehog Pathway by Cyclopamine or GLI1 siRNA Reduces In Vivo Tumorigenesis of Human Medulloblastoma Cells Xenotransplanted to Immunodeficient Nude Mice. Advances Transl Med Res 1(1):1-5

11. Zhang X, Harrington N, Moraes RC, et al. (2009) Cyclopamine inhibition of human breast cancer cell growth independent of Smoothened (Smo). Breast Cancer Res Treat 115: 505-521.

12. Lee ST, Welch KD, Panter KE, et al. (2014) Cyclopamine: from cyclops lambs to cancer treatment. J Agric Food Chem 62: 7355-7362.

13. Axelson M, Liu K, Jiang X, et al. (2013) U.S. Food and Drug Administration approval: vismodegib for recurrent, locally advanced, or metastatic basal cell carcinoma. Clin Cancer Res 19: 2289-2293.

14. Wu C, Hu S, Cheng J, et al. (2017) Smoothened antagonist GDC-0449 (Vismodegib) inhibits proliferation and triggers apoptosis in colon cancer cell lines. Exp Ther Med 13: 2529-2536.

15. Singh BN, Fu J, Srivastava RK, et al. (2011) Hedgehog Signaling Antagonist GDC-0449 (Vismodegib) Inhibits Pancreatic Cancer Stem Cell Characteristics: Molecular Mechanisms. PLoS One 6: e27306.

16. Gajjar A, Stewart CF, Ellison DW, et al. (2013) Phase-I study of vismodegib in children with recurrent or refractory medulloblastoma: a Pediatric Brain Tumor Consortium study (PBTC). Clin Cancer Res 19: 6305-6312.

17. Brinkhuizen T, Reinders MG, van Geel M, et al. (2014) Acquired resistance to the Hedgehog pathway inhibitor vismodegib due to smoothened mutations in treatment of locally advanced basal cell carcinoma. J Am Acad Dermatol 71: 1005-1008.

18. Pricl S, Cortelazzi B, Dal Col V, et al. (2015) Smoothened (SMO) receptor mutations dictate resistance to vismodegib in basal cell carcinoma. Mol Oncol 9: 389-397.
19. Proctor AE, Thompson LA, O'Bryant CL (2014) Vismodegib: an inhibitor of the Hedgehog signaling pathway in the treatment of basal cell carcinoma. Ann Pharmacother 48: 99-106.

20. Ridky TW, Cotsarelis G (2015) Vismodegib resistance in basal cell carcinoma: not a smooth fit. Cancer Cell 27: 315-316.

21. Casey D, Demko S, Shord S, et al. (2017) FDA Approval Summary: Sonidegib for Locally Advanced Basal Cell Carcinoma. Clin Cancer Res 23: 2377-2381.

22. Collier NJ, Ali FR, Lear JT (2016) The safety and efficacy of sonidegib for the treatment of locally advanced basal cell carcinoma. Expert Rev Anticancer Ther 16: 1011-1018.

23. Doan HQ, Silapunt S, Migden MR (2016) Sonidegib, a novel smoothened inhibitor for the treatment of advanced basal cell carcinoma. Onco Targets Ther 9: 5671-5678.

24. Ramelyte E, Amann VC, Dummer R (2016) Sonidegib for the treatment of advanced basal cell carcinoma. Expert Opin Pharmacother 17: 1963-1968.

25. Danial C, Sarin KY, Oro AE, et al. (2016) An Investigator-Initiated Open-Label Trial of Sonidegib in Advanced Basal Cell Carcinoma Patients Resistant to Vismodegib. Clin Cancer Res 22: 1325-1329.

26. Infante P, Alfonsi R, Ingallina C, et al. (2016) Inhibition of Hedgehog-dependent tumors and cancer stem cells by a newly identified naturally occurring chemotype. Cell Death Dis 7: e2376.

27. Tang JY, Xiao TZ, Oda Y, et al. (2011) Vitamin D3 inhibits hedgehog signaling and proliferation in murine Basal cell carcinomas. Cancer Prev Res (Phila) 4: 744-751.

28. Kim DJ, Kim J, Spaunhurst K, et al. (2014) Open-label, exploratory phase II trial of oral itraconazole for the treatment of basal cell carcinoma. J Clin Oncol 32: 745-751. 